\section{Heart Drug"}

AMIS Plus Investigators 131

Andersen Schmidt, T. 63

Andersen, C.B. 146

Andersen, P.S. 54

Balaji, M. 183

Balaji, V. 183

Bech, J. 63, 153

Benatar, V. 21

Bouly, M. 14

Burger, P.C. 75

Cannizzaro, S. 187

Carstensen, S. 101

Chen, M.-Z. 140

Chen, X. 140, 146

Chezaubernard, C. 14

Christiansen, M. 54

Cocco, G. 68

Cotter, G. 161

Di Pasquale, P. 187

Ducharme, A. 214

Erne, P. 131

Fang, C.-H. 140

Fasullo, S. 187

Fujii, K. 193

Giambanco, F. 187

Grande, P. 177

Gutzwiller, F. 121, 131

Halkin, A. 168

Haunsø, S. 146

Hilleman, D.E. 81

Hori, M. 193

Johnsen, H.E. 177

Jørgensen, E. 177

Kääb, S. 51

Kaiser, W.E. 121
Kaluski, E. 161

Kanters, J.K. 54

Kastrup, J. 177

Kinjo, K. 193

Kjeldsen, K. 54, 63, 153

Kodama, K. 193

Krishna Kishore, P.V. 183

Kusuoka, H. 193

Larsen, L.A. 54

Lenz, T.L. 81

Lerebours, G. 14

Lerebours Pigeonnière, G. 21

Li, J.-J. 140

Lundkvist, L. 54

Mahlberg-Gaudin, F.H. 14

Mahler, F. 121

Margitich, V.M. 197

März, W. 39

Masoudi, F.A. 89

Melnyk, V.S. 197

Milo, O. 161

Mishima, M. 193

Mizuno, H. 193

Nakatani, D. 193

Nielsen, H.B. 146

Noseda, G. 131

Nury-Philémon, P. 21

Osaka Acute Coronary Insufficiency Study (OACIS) Group 193

Palardy, M. 214

Pandolfi, S. 68

Paterna, S. 187

Peterson, P.N. 89

Prabhakar, V.R. 100, 183

Radovanovic, D. 131

Ripa, R.S. 177

Rousson, V. 68
Sakata, Y. 193

Sato, H. 193

Scalzo, S. 187

Schilling, J. 131

Schmid, J.-P. 220

Schmidt, T.A. 153

Schoeberlein, A. 75

Schroeder, V. 220

Seifert, B. 75

Seshaiah, V. 183

Shimizu, M. 193

Søndergaard, L. 146

Stone, G.W. 168

Stoschitzky, K. 1, 6

Svendsen, J.H. 146

Szucs, T.D. 121

Tanouchi, J. 193

Tardif, J.-C. 25

Thomsen, C. 146

Toft, B.G. 146

Tonstad, S. 29

Tran, C.T. 54

Tricoli, G. 187

Uriel, N. 161

Vered, Z. 161

Vinychuk, S.M. 197

Vintila, M.M. 11

von Planta, M. 205

Vuust, J. 54

Wang, Y. 177

Wascher, T.C. 34

Wehling, M. 2

Wettrell, G. 54

Zund, G. 75

\title{
KARGER
}

(c) 2005 S. Karger AG, Basel

Fax +4161306 1234 